\title{
MULTIPLIERS AND OPERATORS ON THE TEMPERED ULTRADISTRIBUTION SPACES OF ROUMIEU TYPE FOR THE DISTRIBUTIONAL HANKEL-TYPE TRANSFORMATION SPACES
}

\author{
P. K. BANERJI AND S. K. AL-OMARI \\ Received 4 May 2006; Accepted 4 May 2006
}

The tempered ultradistribution space of Roumieu type for the space $H_{\mu, v}$ is defined, which is a subspace of the Hausdörff locally convex topological linear space. Further, results are obtained for the multipliers and operators on the tempered ultradistribution spaces for the distributional Hankel-type transformation spaces.

Copyright (c) 2006 Hindawi Publishing Corporation. All rights reserved.

\section{Introduction}

During the course of time researchers have defined ultradistributions as the duals of various types of ultradifferentiable functions of Roumieu type and of Buerling type, Roumieu type ultradifferentiable functions have been considered in the present paper. Three types of spaces of rapidly decreasing ultradifferentiable functions of Roumieu type have been defined, which can be considered as subspaces of the Hausdörff locally convex topological linear space $H_{\mu, \nu}$ (cf. [3]), as the set of all infinitely differentiable complex-valued functions $\varphi(x)$ on an interval $I(0, \infty)$ with the norm

$$
\Gamma_{i, j}^{\mu, \nu}(\varphi)=\sup _{x \in I}\left|x^{i}\left(x^{-1} D\right)^{j} x^{-\mu-\nu-1} \varphi(x)\right|<\infty,
$$

where $i, j$ are a pair of nonnegative integers and $\mu, v$ are real numbers which will be an auxiliary to obtain duals of Roumieu-type tempered ultradistributions.

The $a_{i} s$ and $b_{j} s$, wherever they appear $(i, j=0,1,2, \ldots)$, are to be considered as sequences of positive real numbers on which the constraints imposed are (cf. [2, page 66])

$$
\begin{array}{ll}
a_{i}^{2} \leq a_{i-1} a_{i+1} & \forall i \in \mathbb{N}, \\
b_{j}^{2} \leq b_{j-1} b_{j+1} & \forall j \in \mathbb{N} .
\end{array}
$$

$S, S_{1}>0$ and $T, T_{1}>1$ are constants such that

$$
\begin{aligned}
& a_{i} \leq S T^{i} \min _{0 \leq k \leq i} a_{k} a_{i-k}, \quad i \in \mathbb{N}_{0}, \\
& b_{j} \leq S_{1} T_{1}^{j} \min _{0 \leq k \leq j} b_{k} b_{j-k}, \quad j \in \mathbb{N}_{0} .
\end{aligned}
$$

Hindawi Publishing Corporation

International Journal of Mathematics and Mathematical Sciences

Volume 2006, Article ID 31682, Pages 1-7

DOI 10.1155/IJMMS/2006/31682 
2 Tempered ultradistribution space of Roumieu type

As a consequence of (1.2), we have (cf. [1])

$$
\begin{aligned}
& a_{i} a_{k} \leq a_{0} a_{i+k}, \quad i, k \in \mathbb{N}_{0}, \\
& b_{j} b_{k} \leq b_{0} b_{j+k}, \quad j, k \in \mathbb{N}_{0} .
\end{aligned}
$$

Definition 1.1. (I) $H_{\mu, a_{i}, A}^{v}$ is the space of all those functions, defined on interval $I$, and are infinitely smooth, for which

$$
\left|x^{i}\left(x^{-1} D\right)^{j} x^{-\mu-\nu-1} \varphi(x)\right| \leq C_{j}^{\mu, \nu}(A+\alpha)^{i} a_{i}, \quad i, j \in \mathbb{N}_{0},
$$

where $A$ and $C_{j}^{\mu, \nu}$ are constants depending on $\varphi$ and $\alpha$ is an arbitrary positive constant.

(II) Define $\varphi \in H_{\mu}^{v, b_{j}, B}$ whenever $\varphi$ is smooth and the inequality

$$
\left|x^{i}\left(x^{-1} D\right)^{j} x^{-\mu-\nu-1} \varphi(x)\right| \leq C_{j}^{\mu, \nu}(B+\beta)^{j} b_{j}
$$

is true for all $i, j \in \mathbb{N}_{0}$.

(III) For $\varphi$ being a smooth function, $\varphi(x) \in H_{\mu, a_{i}, A}^{v, b_{j}, B}$, which satisfies the inequality

$$
\left|x^{i}\left(x^{-1} D\right)^{j} x^{-\mu-\nu-1} \varphi(x)\right| \leq C^{\mu, \nu}(A+\alpha)^{i}(B+\beta)^{j} a_{i} b_{j}
$$

where $i, j \in \mathbb{N}_{0}$ (arbitrary constants) and $\alpha, \beta>0, A, B$, and $C^{\mu, v}$ are positive constants depending on $\varphi$.

The norms on the spaces $H_{\mu, a_{i}, A}^{v}, H_{\mu}^{v, b_{j}, B}$, and $H_{\mu, a_{i}, A}^{v, b_{j}, B}$ are defined as

$$
\begin{aligned}
& \vartheta_{j, \alpha}^{\mu, v}(\varphi)=\sup _{\substack{x \in(0, \infty) \\
i \in \mathbb{N}_{0}}} \frac{\left|x^{i}\left(x^{-1} D\right)^{j} x^{-\mu-v-1} \varphi(x)\right|}{(A+\alpha)^{i} a_{i}}, \\
& \vartheta_{i, \beta}^{\mu, v}(\varphi)=\sup _{\substack{x \in(0, \infty) \\
i \in \mathbb{N}_{0}}} \frac{\left|x^{i}\left(x^{-1} D\right)^{j} x^{-\mu-v-1} \varphi(x)\right|}{(B+\beta)^{j} b_{j}},
\end{aligned}
$$

where $i, j=0,1,2,3, \ldots$ and $\alpha, \beta=1,1 / 2,1 / 3, \ldots$, and

$$
\vartheta_{\alpha, \beta}^{\mu, v}(\varphi)=\sup _{\substack{x \in(0, \infty) \\ i, j \in \mathbb{N}_{0}}} \frac{\left|x^{i}\left(x^{-1} D\right)^{j} x^{-\mu-v-1} \varphi(x)\right|}{(A+\alpha)^{i}(B+\beta)^{j} a_{i} b_{j}}
$$

for the conditions already mentioned.

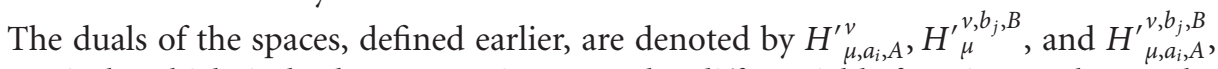
respectively, which, indeed, are Roumieu-type ultradifferentiable functions. Whereas the duals are the tempered ultradistributions of Roumieu type.

The spaces $H_{\mu, a_{i}, A}^{v}, H_{\mu}^{v, b_{j}, B}$, and $H_{\mu, a_{i}, A}^{v, b_{j}, B}$ being the subspaces of the space $H_{\mu, v}$, indeed, $H_{\mu, v}^{\prime}$ will be a subspace of their duals. 


\section{Multipliers for ultradifferentiable functions}

This section deals with results, which justify certain mapping to be linear on the three spaces defined earlier. The following definition will make sense in the analysis of the results.

Definition 2.1. Denote by $\mathscr{E}_{a_{i}, A_{0}}, \mathscr{E}^{b_{j}, B_{0}}$, and $\mathscr{E}_{a_{i}, A_{0}}^{b_{j}, B_{0}}$ the set of all complex-valued infinitely differentiable functions ( $C^{\infty}$-functions) $\theta(x)$ on the interval $(0, \infty)$, which satisfy the following:

$$
\begin{aligned}
& \left|\left(x^{-1} D\right)^{i} \theta(x)\right| \leq C A_{0}^{i} a_{i}\left(1+x^{r}\right), \\
& \left|\left(x^{-1} D\right)^{j} \theta(x)\right| \leq C B_{0}^{j} b_{j}\left(1+x^{r}\right), \\
& \left|\left(x^{-1} D\right)^{j} \theta(x)\right| \leq E A_{0}^{i} B_{0}^{j} a_{j} b_{j}\left(1+x^{r}\right),
\end{aligned}
$$

where $C, D, E$ are positive constants and $r>0$ is an arbitrary integer.

Theorem 2.2. Let $\varphi \in H_{\mu, a_{i}, A}^{v}, H_{\mu}^{v, b_{j}, B}$, and $H_{\mu, a_{i}, A}^{v, b_{j}, B}$, respectively. Then

$$
\begin{gathered}
\vartheta_{j, 1 / n}^{\mu, \nu}(\varphi) \leq \vartheta_{j, 1 /(n+1)}^{\mu, \nu}(\varphi), \quad \vartheta_{i, 1 / n}^{\mu, \nu}(\varphi) \leq \vartheta_{i, 1 /(n+1)}^{\mu, \nu}(\varphi), \\
\vartheta_{1 / n}^{\mu, \nu}(\varphi) \leq \vartheta_{1 /(n+1), 1 /(n+1)}^{\mu, \nu}(\varphi), \quad \text { respectively. }
\end{gathered}
$$

Proofs of the above inequalities are straightforward consequences of (1.8) and (1.9), respectively. The details are thus avoided.

Theorem 2.3. Let $\theta \in \mathscr{E}_{a_{i}, A_{0}}$ and $\varphi \in H_{\mu, a_{i}, A}^{v}$. Then

$$
\vartheta_{j, \alpha}^{\mu, v}(\theta \varphi) \leq 2 C A_{0}^{j} a_{j} \sum_{k=0}^{j}\left(\begin{array}{l}
j \\
k
\end{array}\right) \vartheta_{j-k, \alpha}^{\mu, v}(\varphi),
$$

that is, the mapping $\varphi \rightarrow \theta \varphi$ is a continuous linear mapping on $H_{\mu, a_{i}, A}^{v}$ into itself.

Proof. By virtue of Leibniz theorem and employing (1.5) and Definition 2.1, we obtain

$$
\begin{aligned}
& \left|x^{i}\left(x^{-1} D\right)^{j} x^{-\mu-\nu-1}(\theta \varphi)(x)\right| \\
& =\left|\sum_{k=0}^{j}\left(\begin{array}{l}
j \\
k
\end{array}\right) \frac{\left(x^{-1} D\right)^{k} \theta(x)}{\left(1+x^{r}\right)} x^{i}\left(1+x^{r}\right)\left(x^{-1} D\right)^{j-k} x^{-\mu-\nu-1} \varphi(x)\right| \\
& \leq \sum_{k=0}^{j}\left(\begin{array}{l}
j \\
k
\end{array}\right) C A_{0}^{j} a_{j}\left\{\left|x^{i}\left(x^{-1} D\right)^{j-k} x^{-\mu-\nu-1} \varphi(x)\right|\right. \\
& \left.+\left|x^{i+r}\left(x^{-1} D\right)^{j-k} x^{-\mu-\nu-1} \varphi(x)\right|\right\} .
\end{aligned}
$$




\section{Tempered ultradistribution space of Roumieu type}

By multiplying by $1 /(A+\alpha)^{i} a_{i}$ and considering the supremum over all integers $i>0$, for all $x \in(0, \infty)$, the above relation is expressed as

$$
\begin{aligned}
& \sup _{\substack{i \in \mathbb{N}_{0} \\
x \in(0, \infty)}} \frac{\left|x^{i}\left(x^{-1} D\right)^{j} x^{-\mu-v-1}(\theta \varphi)(x)\right|}{(A+\alpha)^{i} a_{i}} \\
& \leq \sum_{k=0}^{j}\left(\begin{array}{l}
j \\
k
\end{array}\right) C A_{0}^{j} a_{j}\left[\sup _{\substack{i \in \mathbb{N}_{0} \\
x \in(0, \infty)}} \frac{\left|x^{i}\left(x^{-1} D\right)^{j-k} x^{-\mu-v-1} \varphi(x)\right|}{(A+\alpha)^{i} a_{i}}\right. \\
&\left.+\sup _{\substack{i \in \mathbb{N}_{0} \\
x \in(0, \infty)}} \frac{\left|x^{i+v}\left(x^{-1} D\right)^{j-k} x^{-\mu-v-1} \varphi(x)\right|}{(A+\alpha)^{i} a_{i}}\right],
\end{aligned}
$$

that is,

$$
\vartheta_{j, \alpha}^{\mu, v}(\theta \varphi) \leq C A_{0}^{j} a_{j} \sum_{k=0}^{j}\left(\begin{array}{l}
j \\
k
\end{array}\right) \vartheta\left(j-k, \alpha^{\mu, v}(\varphi)+\right) \vartheta_{j-k, \alpha}^{\mu, v}(\varphi)
$$

that is,

$$
\vartheta_{j, \alpha}^{\mu, v}(\theta \varphi) \leq 2 C A_{0}^{j} a_{j} \sum_{k=0}^{j}\left(\begin{array}{l}
j \\
k
\end{array}\right) \vartheta_{j-k, \alpha}^{\mu, v}(\varphi) .
$$

This completes the proof of the theorem.

THEOREM 2.4. If $\mathscr{E} b_{j}, B_{0}$ and $b_{j}$ satisfy the relation (1.4), then for all $\varphi \in H_{\mu}^{v, b_{j}, B}$, the map $\varphi \rightarrow \theta \varphi$ is a continuous linear map on the space $H_{\mu}^{v, b_{j}, B}$ into itself, that is,

$$
\vartheta_{j, \alpha}^{\mu, v}(\theta \varphi) \leq 2 D b_{0} B_{0}^{j} b_{j} \sum_{k=0}^{j}\left(\begin{array}{l}
j \\
k
\end{array}\right) \vartheta_{j-k, \beta}^{\mu, v}(\varphi)
$$

Proof. Employing Definition 2.1 to (2.8) and multiplying it by $1 /(B+\beta)^{j} b_{j}$, we have

$$
\begin{aligned}
& \frac{\left|x^{i}\left(x^{-1} D\right)^{j} x^{-\mu-\nu-1}(\theta \varphi)(x)\right|}{(B+\beta)^{j} b_{j}} \\
& \quad \leq \sum_{k=0}^{j}\left(\begin{array}{l}
j \\
k
\end{array}\right) D B_{0}^{j} b_{j}\left(\left|x^{i}\left(x^{-1} D\right)^{j-k} x^{-\mu-\nu-1} \varphi(x)\right|\right. \\
& \left.\quad+\left|x^{i+v}\left(x^{-1} D\right)^{j-k} x^{-\mu-\nu-1} \varphi(x)\right|\right) \frac{1}{(B+\beta)^{j} b_{j}} .
\end{aligned}
$$

Now by virtue of (1.4), $1 / b_{j} \leq b_{0} / b_{k} b_{j-k}$. Owing to $j-k>0, x^{i} \leq x^{i+r}$ for all $i, r>0$, 
from (2.9) we have

$$
\begin{aligned}
& \frac{\left|x^{i}\left(x^{-1} D\right)^{j} x^{-\mu-\nu-1}(\theta \varphi)(x)\right|}{(B+\beta)^{j} b_{j}} \\
& \leq \sum_{k=0}^{j}\left(\begin{array}{l}
j \\
k
\end{array}\right) D B_{0}^{j} \frac{b_{j} b_{0}}{b_{k}(B+\beta)^{k}}\left(\frac{\left|x^{i}\left(x^{-1} D\right)^{j-k} x^{-\mu-\nu-1} \varphi(x)\right|}{(B+\beta)^{j-k} b_{j-k}}\right. \\
&\left.+\frac{\left|x^{i+r}\left(x^{-1} D\right)^{j-k} x^{-\mu-\gamma-1} \varphi(x)\right|}{(B+\beta)^{j-k} b_{j-k}}\right)
\end{aligned}
$$

for all integers $j>0$, and for all $x \in(0, \infty)$, we consider the supremum to obtain

$$
\vartheta_{i, \beta}^{\mu, v}(\theta \varphi) \leq 2 D b_{0} B_{0}^{j} b_{j} \sum_{k=0}^{j}\left(\begin{array}{l}
j \\
k
\end{array}\right) \vartheta_{i+r, \beta}^{\mu, \nu}(\varphi) .
$$

The theorem is thus completely proved.

Theorem 2.5. If $\theta \in \mathscr{C}_{a_{i}, A_{0}}^{b_{j}, B_{0}}, \varphi \in H_{\mu, a_{i}, A}^{v, b_{j}, B}$, and $b_{j}$ satisfy (1.4), then

$$
\vartheta_{i, \beta}^{\mu, v}(\theta \varphi) \leq C \sum_{k=0}^{j} \frac{\left(\begin{array}{l}
j \\
k
\end{array}\right)}{b_{k}} \vartheta_{\alpha, \beta}^{\mu, v}(\varphi)
$$

that is, the mapping $\varphi \rightarrow \theta \varphi$ is a continuos linear mapping on $H_{\mu, a_{i}, A}^{v, b_{j}, B}$ into itself, where $C=$ $E A_{0}^{j} B_{0}^{j} a_{i} b_{j} b_{0}$.

Proof. By virtue of (2.8) and Definition 1.1, we are led to

$$
\begin{aligned}
& \left|x^{i}\left(x^{-1} D\right)^{j} x^{-\mu-\nu-1}(\theta \varphi)(x)\right| \\
& \quad \leq \sum_{k=0}^{j}\left(\begin{array}{l}
j \\
k
\end{array}\right) E A_{0}^{j} B_{0}^{j} a_{j} b_{j}\left|\left(x^{i}+x^{i+r}\right)\left(x^{-1} D\right)^{j-k} x^{-\mu-\nu-1} \varphi(x)\right| .
\end{aligned}
$$

Now multiplying this relation by $1 /(A+\alpha)^{i}(B+\beta)^{j} a_{i} b_{j}$, we conclude, indeed, that $1 /(B+$ $\beta)^{k} \leq 1$, that is,

$$
\begin{aligned}
& \frac{\left|x^{i}\left(x^{-1} D\right)^{j} x^{-\mu-\nu-1}(\theta \varphi)(x)\right|}{(A+\alpha)^{i}(B+\beta)^{j} a_{i} b_{j}} \\
& \quad \leq \sum_{k=0}^{j}\left(\begin{array}{l}
j \\
k
\end{array}\right) E A_{0}^{j} B_{0}^{j} a_{j} b_{j} \frac{b_{j-k}}{b_{j}}\left|\frac{\left(x^{i}+x^{i+r}\right)\left(x^{-1} D\right)^{j-k} x^{-\mu-\nu-1} \varphi(x)}{(A+\alpha)^{i}(B+\beta)^{j-k} a_{i} b_{j-k}}\right| .
\end{aligned}
$$


6 Tempered ultradistribution space of Roumieu type

Further, employing (1.4) and as usual taking the supremum over all $x \in(0, \infty), i, j \in \mathbb{N}_{0}$, we have

$$
\vartheta_{i, \beta}^{\mu, v}(\theta \varphi) \leq C \sum_{k=0}^{j} \frac{\left(\begin{array}{l}
j \\
k
\end{array}\right)}{b_{k}} \vartheta_{\alpha, \beta}^{\mu, v}(\varphi),
$$

where $C$ has the usual meaning. Thus, the theorem is completely proved.

\section{Operators for the duals of tempered ultradistribution}

In this section, a lemma is proved to establish the isomorphism between the spaces which are infinitely smooth and defined on $(0, \infty)$, described in Section 1 and further, a mapping is proved to be an isomorphism between duals of these spaces.

Lemma 3.1. For $k$ being a nonnegative integer, the mapping $\varphi \rightarrow x^{k} \varphi$ is an isomorphism from the spaces $H_{\mu, a_{i}, A}^{v}, H_{\mu}^{v, b_{j}, B}$, and $H_{\mu, a_{i}, A}^{v, b_{j}, B}$ onto $H_{\mu, a_{i}, A}^{v+k}, H_{\mu}^{v+k, b_{j}, B}$, and $H_{\mu, a_{i}, A}^{v, b_{j}, B}$, respectively.

Proof. Let $\varphi \in H_{\mu, a_{i}, A}^{v}, H_{\mu}^{v, b_{j}, B}$, and $H_{\mu, a_{i}, A}^{v, b_{j}, B}$, respectively.

In the case $\varphi \in H_{\mu, a_{i}, A}^{v}$, we write

$$
\left|x^{i}\left(x^{-1} D\right)^{j} x^{-\mu-(\nu+k)-1}\left(x^{k} \varphi(x)\right)\right|=\left|x^{i}\left(x^{-1} D\right)^{j} x^{-\mu-\nu-1} \varphi(x)\right| .
$$

Now multiplying (3.1) by $1 /(A+\alpha)^{i} a_{i}$ and taking supremum over all $x \in(0, \infty)$, for $j \in$ $\mathbb{N}_{0}$, we obtain

$$
\vartheta_{j, \alpha}^{\mu, v+k}\left(x^{k} \varphi\right)=\vartheta_{j, \alpha}^{\mu, v}(\varphi)
$$

Now, when $\varphi \in H_{\mu}^{v, b_{j}, B}$, we multiply (3.1) by $1 /(B+\beta)^{j} b_{j}$, and following the above lines of the proof, we arrive at

$$
\vartheta_{i, \beta}^{\mu, v+k}\left(x^{k} \varphi\right)=\vartheta_{i, \beta}^{\mu, v}(\varphi)
$$

Finally, for the case $\varphi \in H_{\mu, a_{i}, A}^{v, b_{j}, B}$, similarly, we conclude to write

$$
\vartheta_{\alpha, \beta}^{\mu, v+k}\left(x^{k} \varphi\right)=\vartheta_{\alpha, \beta}^{\mu, v}(\varphi) .
$$

The lemma is completely proved.

As an obvious consequence of Lemma 3.1 and the result (cf. [4, Theorem 1.10-2, page 29]), for $k \geq 0$, it is shown that the mapping $f \rightarrow x^{k} f$ is an isomorphism from the duals $H_{\mu, a_{i}, A}^{\prime v+k},{H^{\prime}}_{\mu}^{v+k, b_{j}, B}$, and ${H^{\prime}}_{\mu, a_{i}, A}^{\nu+k, b_{j}, B}$, respectively, onto $H_{\mu, a_{i}, A}^{\prime v},{H^{\prime}}_{\mu}^{v, b_{j}, B}$, and ${H^{\prime}}_{\mu, a_{i}, A}^{\nu, b_{j}, B}$, respectively.

\section{Acknowledgment}

The second author (SKA) acknowledges the assistance of the JRF of UGC (India) sanctioned to him, which partially supports this work (Sanction no. F.19-1/ 2004 (SA-II)). 


\section{References}

[1] R. D. Carmichael, R. S. Pathak, and S. Pilipović, Cauchy and Poisson integrals of ultradistributions, Complex Variables 14 (1990), no. 1-4, 85-108.

[2] H. Komatsu, Ultradistributions. I: structure theorems and a characterization, Journal of the Faculty of Science. University of Tokyo. Section I A. Mathematics 20 (1973), 25-105.

[3] M. Linares Linares and J. M. R. Méndez Pérez, A Hankel type integral transformation on certain space of distributions, Bulletin of the Calcutta Mathematical Society 83 (1991), no. 5, 447-456.

[4] A. H. Zemanian, Generalized Integral Transformations, Dover, New York, 1987, first published by Interscience, New York, 1968.

P. K. Banerji: Department of Mathematics, Faculty of Science, JNV University, Jodhpur 342005, India

E-mail address: banerjipk@yahoo.com

S. K. Al-Omari: Department of Mathematics, Faculty of Science, JNV University, Jodhpur 342005, India

E-mail address: shrideh2001@yahoo.co.in 


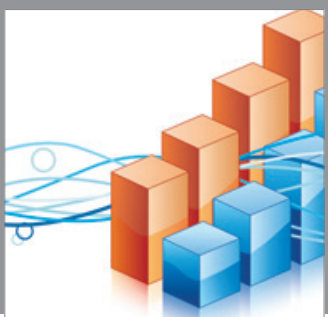

Advances in

Operations Research

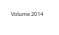

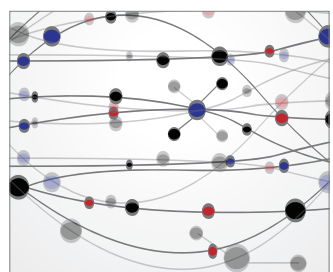

\section{The Scientific} World Journal
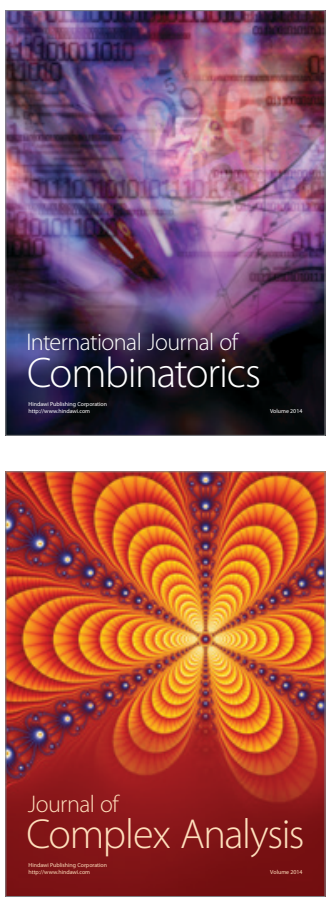

International Journal of

Mathematics and

Mathematical

Sciences
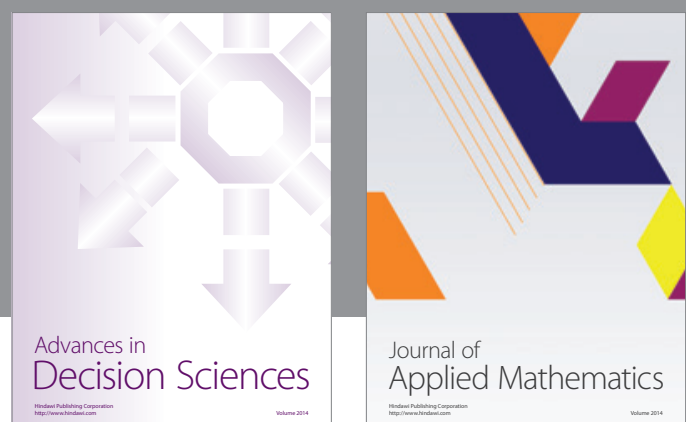

Journal of

Applied Mathematics
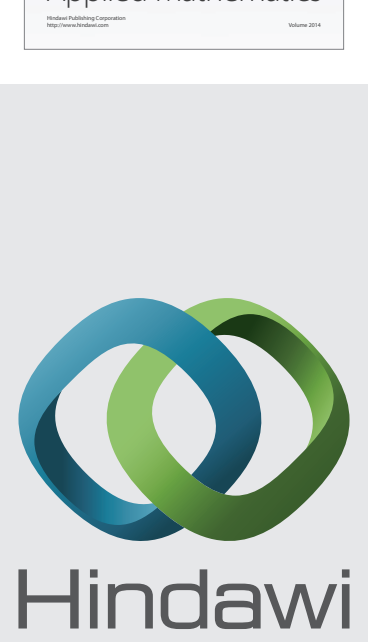

Submit your manuscripts at http://www.hindawi.com
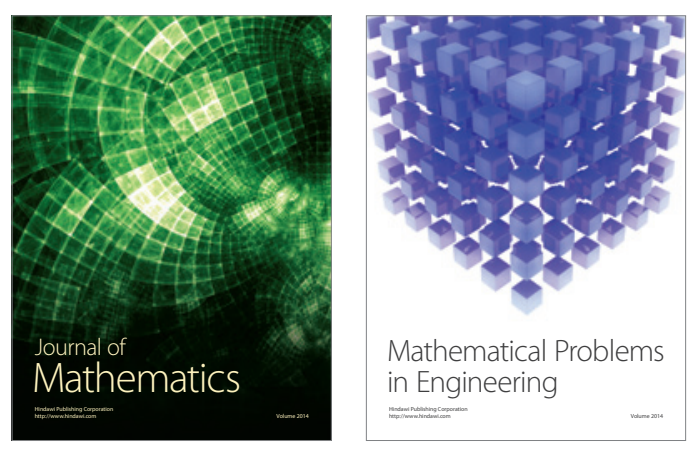

Mathematical Problems in Engineering
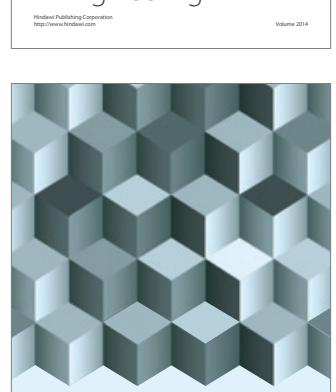

Journal of

Function Spaces
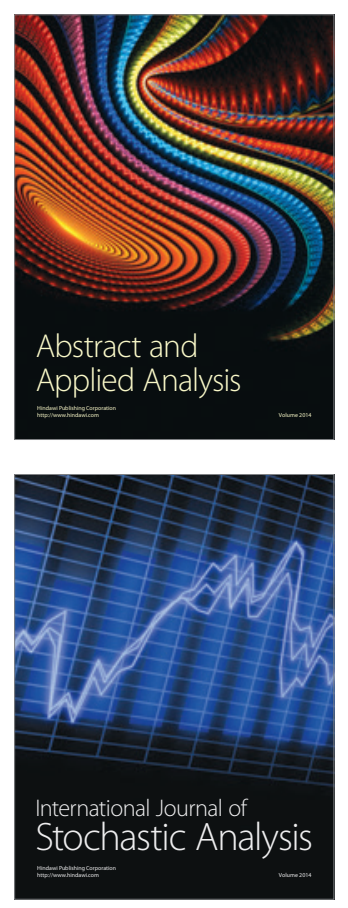

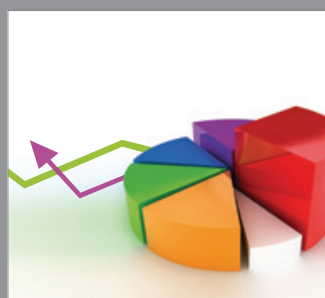

ournal of

Probability and Statistics

Promensencen
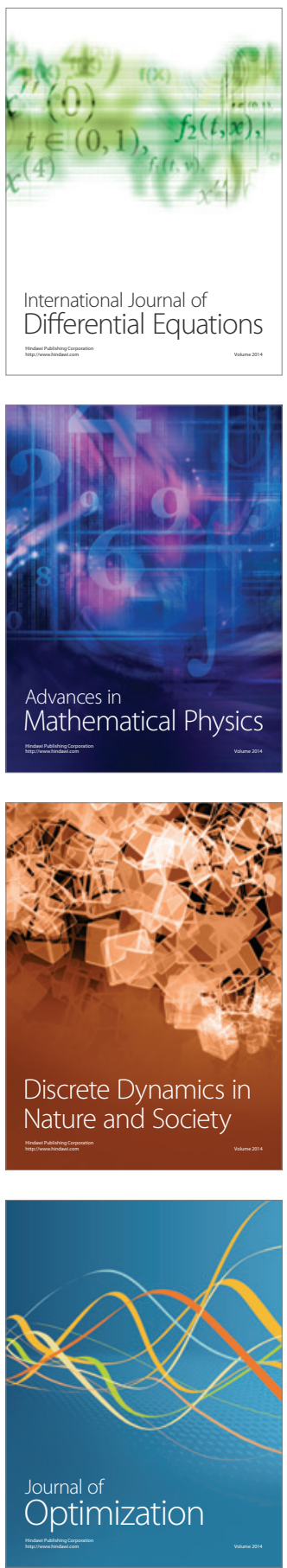